\title{
The genome sequence of the grey wolf, Canis lupus Linnaeus
}

\section{8 [version 1; peer review: 2 approved]}

\section{Mikkel-Holger S. Sinding (Di)1,2, Shyam Gopalakrishnan1,3, Katrine Raundrup (iD), Love Dalén ${ }^{4-6}$, Jonathan Threlfall (D7, Darwin Tree of Life Barcoding collective, Wellcome Sanger Institute Tree of Life programme, Wellcome Sanger Institute Scientific Operations: DNA Pipelines collective, Tree of Life Core Informatics collective, Darwin Tree of Life Consortium, Tom Gilbert (D1,3,8}

\footnotetext{
${ }^{1}$ Globe Institute, University of Copenhagen, Copenhagen, Denmark

${ }^{2}$ Greenland Institute of Natural Resources, Kivioq 2, Nuuk, Greenland

${ }^{3}$ Center for Evolutionary Hologenomics, University of Copenhagen, Copenhagen, Denmark

${ }^{4}$ Centre for Palaeogenetics, Stockholm, Sweden

${ }^{5}$ Department of Bioinformatics and Genetics, Swedish Museum of Natural History, Stockholm, Sweden

${ }^{6}$ Department of Zoology, Stockholm University, Stockholm, Sweden

${ }^{7}$ Tree of Life, Wellcome Sanger Institute, Cambridge, UK

${ }^{8}$ Norwegian University of Science and Technology, Trondheim, Norway
}

V1 First published: 12 Nov 2021, 6:310

https://doi.org/10.12688/wellcomeopenres.17332.1

Latest published: 12 Nov 2021, 6:310

https://doi.org/10.12688/wellcomeopenres.17332.1

\section{Abstract}

We present a genome assembly from an individual male Canis lupus orion (the grey wolf, subspecies: Greenland wolf; Chordata;

Mammalia; Carnivora; Canidae). The genome sequence is 2,447

megabases in span. The majority of the assembly $(98.91 \%)$ is scaffolded into 40 chromosomal pseudomolecules, with the $X$ and $Y$ sex chromosomes assembled.

\section{Keywords}

Canis lupus, Canis lupus orion, grey wolf, Polar wolf, Greenland wolf, genome sequence, chromosomal

This article is included in the Tree of Life

gateway.

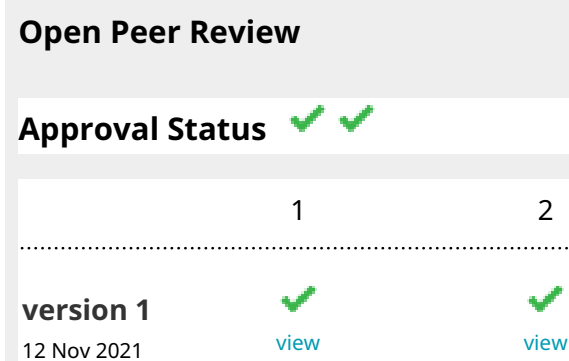

1. Michael Hiller (D), LOEWE Centre for Translational Biodiversity Genomics, Frankfurt am Main, Germany Senckenberg Society for Nature Research, Frankfurt am Main, Germany Goethe University, Frankfurt am Main, Germany

2. Rémi Allio, University of Montpellier, Montpellier, France

Any reports and responses or comments on the article can be found at the end of the article. 
Corresponding author: Darwin Tree of Life Consortium (mark.blaxter@sanger.ac.uk)

Author roles: Sinding MHS: Investigation, Methodology, Resources, Writing - Original Draft Preparation, Writing - Review \& Editing; Gopalakrishnan S: Investigation, Resources, Writing - Review \& Editing; Raundrup K: Investigation, Methodology, Resources, Writing Review \& Editing; Dalén L: Investigation, Methodology, Resources, Writing - Review \& Editing; Threlfall J: Project Administration, Writing - Original Draft Preparation, Writing - Review \& Editing; Gilbert T: Investigation, Resources, Supervision, Writing - Review \& Editing

Competing interests: Jonathan Threlfall was employed by F1000 Research Ltd until January 2021.

Grant information: This work was supported by Wellcome through core funding to the Wellcome Sanger Institute (206194) and the Darwin Tree of Life Discretionary Award (218328). The authors acknowledge support from the National Genomics Infrastructure in Stockholm funded by Science for Life Laboratory, the Knut and Alice Wallenberg Foundation and the Swedish Research Council, and SNIC/Uppsala Multidisciplinary Center for Advanced Computational Science for assistance with massively parallel sequencing and access to the UPPMAX computational infrastructure.

The funders had no role in study design, data collection and analysis, decision to publish, or preparation of the manuscript.

Copyright: (C) 2021 Sinding MHS et al. This is an open access article distributed under the terms of the Creative Commons Attribution License, which permits unrestricted use, distribution, and reproduction in any medium, provided the original work is properly cited.

How to cite this article: Sinding MHS, Gopalakrishnan S, Raundrup K et al. The genome sequence of the grey wolf, Canis Iupus Linnaeus 1758 [version 1; peer review: 2 approved] Wellcome Open Research 2021, 6:310 https://doi.org/10.12688/wellcomeopenres.17332.1

First published: 12 Nov 2021, 6:310 https://doi.org/10.12688/wellcomeopenres.17332.1 


\section{Species taxonomy}

Eukaryota; Metazoa; Chordata; Craniata; Vertebrata; Euteleostomi; Mammalia; Eutheria; Laurasiatheria; Carnivora; Caniformia; Canidae; Canis; Canis lupus Linnaeus 1758 (NCBI:txid9612).

\section{Background}

The grey wolf, Canis lupus, is the largest species within the group wolf-like canids (Subtribe: Canina) and the member with the largest geographic distribution. Originally wolves were found throughout Eurasia, with the exception of tropical Southeast Asia, and all of North America. This vast distribution contains numerous habitats, encompassing wolf ecotypes adapted to the diverse environments throughout their distribution. The wolf is locally extinct in several places, such as the UK, Ireland and Brittany, yet it still holds much of its original distribution; the global population is estimated to be in the order of 200-250 thousand individuals (Jhala et al., 2018).

Once numerous, wolves were eradicated from the islands of Great Britain in the 15th century and Ireland in the 18th century. There have been proposals to reintroduce populations of wolves to the Scottish Highlands to manage populations of red deer, which have a negative effect on biodiversity through overgrazing (Nilsen et al., 2007). The Scottish Highlands are considered to be the only location in Great Britain that could support a healthy population of wolves; however, objections of livestock owners are likely to prevent their reintroduction in the near future (Wilson, 2004). The reintroduction of wolves elsewhere has led not only to the reestablishment of this apex predator, but also to marked improvements in biodiversity in the ecosystem as a whole (Ripple et al., 2014). Wolves reintroduced into the Yellowstone National Park, Wyoming, USA, in 1995 predated grazing animals such as wapiti (Cervus canadensis) that preserved grasslands. The subsequent changes in prey behaviour led to trophic cascades that resulted in the reestablishment of tree species and an associated increase in populations of species that rely directly and indirectly on this habitat (Ripple \& Beschta, 2012).

Wolves have historically been found in Northwest, Northeast and East Greenland (Dawes et al., 1986). Wolves were extirpated from East Greenland through hunting by 1939 and were absent from this area for the next 40 years (MarquardPetersen, 2012). In around 1979, a pair of wolves travelled from the north of the island and began a recolonisation of East Greenland, establishing a population of around 23 wolves (Marquard-Petersen, 2011). A recent assessment found no trace of wolves for a number of years in East Greenland, while a population of up to 32 animals is still found in the northernmost parts of Greenland. Since the population in East Greenland was located entirely within the Northeast Greenland National Park, affording the wolves legal protection, it is unlikely that this extinction event was driven by hunting (Marquard-Petersen, 2021).

Domestic dogs share a common ancestor with Eurasian wolves around 33,000 years ago (Skoglund et al., 2015; Wang et al., 2016). In this regard, the Greenland wolf or Polar wolf reference genome described herein is highly relevant for dog and/or Eurasian wolf genomics. The Polar wolf is a North American wolf, an outgroup to dogs and Eurasian wolves
(Gopalakrishnan et al., 2019; Sinding et al., 2018), which will aid in making a minimally reference-biased representation of diversity in re-sequenced genomes (Gopalakrishnan et al., 2017). The Polar wolf is also the North American wolf type with the least coyote-like ancestry (Sinding et al., 2018); thus, it is probably the closest possible outgroup to dogs and Eurasian wolves with the least amount of exotic admixture that other North American wolves carry. Finally, this reference genome permits detailed genomic investigations of Polar wolves themselves, as a precise reference, to identify rare genomic variation. The genome is therefore an overall useful resource for research in the Polar wolf itself, a small, isolated and understudied population, but also canids, wolves and dogs overall.

\section{Genome sequence report}

The genome was sequenced from a single male $C$. lupus subspecies orion collected from Siorapaluk, Greenland (latitude 77.785278, longitude -70.631389) in 2016. A total of 28-fold coverage in Pacific Biosciences single-molecule long reads and 74-fold coverage in $10 \mathrm{X}$ Genomics read clouds were generated. Primary assembly contigs were scaffolded with chromosome conformation Hi-C data. Manual assembly curation corrected 135 missing/misjoins and removed 9 haplotypic duplications, reducing the assembly length by $0.2 \%$ and the scaffold number by $42.1 \%$, and increasing the scaffold N50 by $15.9 \%$.

The final assembly has a total length of $2,447 \mathrm{Mb}$ in 82 sequence scaffolds with a scaffold N50 of $66 \mathrm{Mb}$ (Table 1). Of the assembly sequence, $98.91 \%$ was assigned to 40 chromosomal-level scaffolds (named by synteny to an assembly for C. lupus familiaris, breed labrador: GCF_014441545.1), including 38 autosomes and the $\mathrm{X}$ and $\mathrm{Y}$ chromosomes (Figure 1-Figure 4; Table 2). The assembly has a BUSCO (Simão et al., 2015)

Table 1. Genome data for Canis lupus, mCanLor1.2.

\begin{tabular}{|c|c|}
\hline \multicolumn{2}{|l|}{ Project accession data } \\
\hline Assembly identifier & mCanLor1.2 \\
\hline Species & Canis lupus \\
\hline Specimen & mCanLor1 \\
\hline NCBI taxonomy ID & NCBI:txid9612 \\
\hline BioProject & PRJEB43200 \\
\hline BioSample ID & SAMEA7532739 \\
\hline Isolate information & Male, muscle \\
\hline \multicolumn{2}{|l|}{ Raw data accessions } \\
\hline PacificBiosciences SEQUEL II & $\begin{array}{l}\text { ERR6406204, ERR6406205, } \\
\text { ERR6412029, ERR6412030, } \\
\text { ERR6412359, ERR6412360 }\end{array}$ \\
\hline 10X Genomics Illumina & ERR6054484-ERR6054491 \\
\hline Hi-C Illumina & ERR6511153 \\
\hline Illumina RNA-Seq & ERR6054492 \\
\hline
\end{tabular}

Page 3 of 12 


\begin{tabular}{|l|l|}
\hline $\begin{array}{l}\text { Genome assembly } \\
\text { Assembly accession }\end{array}$ & GCA_905319855.2 \\
\hline $\begin{array}{l}\text { Accession of alternate } \\
\text { haplotype }\end{array}$ & GCA_905319845.1 \\
\hline Span (Mb) & 2,447 \\
\hline Number of contigs & 248 \\
\hline Contig N50 length (Mb) & 34 \\
\hline Number of scaffolds & 82 \\
\hline Scaffold N50 length (Mb) & 66 \\
\hline Longest scaffold (Mb) & 123 \\
\hline BUSCO* genome score & C:95.8\%[S:94.6\%,D:1.2\%], \\
\hline
\end{tabular}

*BUSCO scores based on the carnivora_odb10 BUSCO set using v5.1.2. $C=$ complete $[S=$ single copy, $D=$ duplicated], $F=$ fragmented $M=$ missing, $n=$ number of orthologues in comparison. A full set of BUSCO scores is available at https://blobtoolkit.genomehubs.org/ view/Canis\%20lupus/dataset/CAJNRB02/busco, completeness of $95.5 \%$ (single $93.0 \%$, duplicated $2.4 \%$ ) using the carnivora_odb10 reference set. While not fully phased, the assembly deposited is of one haplotype. Contigs corresponding to the second haplotype have also been deposited.

\section{Methods}

A single 4-year-old male C. lupus orion (mCanLor1) was collected from Siorapaluk, Greenland (latitude 77.785278, longitude -70.631389) by The Ministry of Fisheries, Hunting and Agriculture, Government of Greenland. The animal was put down by the local municipal bailiff in Siorapaluk on 13 January 2016. The wolf had little fear of humans, persistently entered the village and could not be chased away. It was therefore decided that the wolf should be killed to protect villagers and dogs in Siorapaluk. After termination, the skull of the specimen was confiscated by the authorities and made available for the purposes of research to the Greenland Institute of Natural Resources.

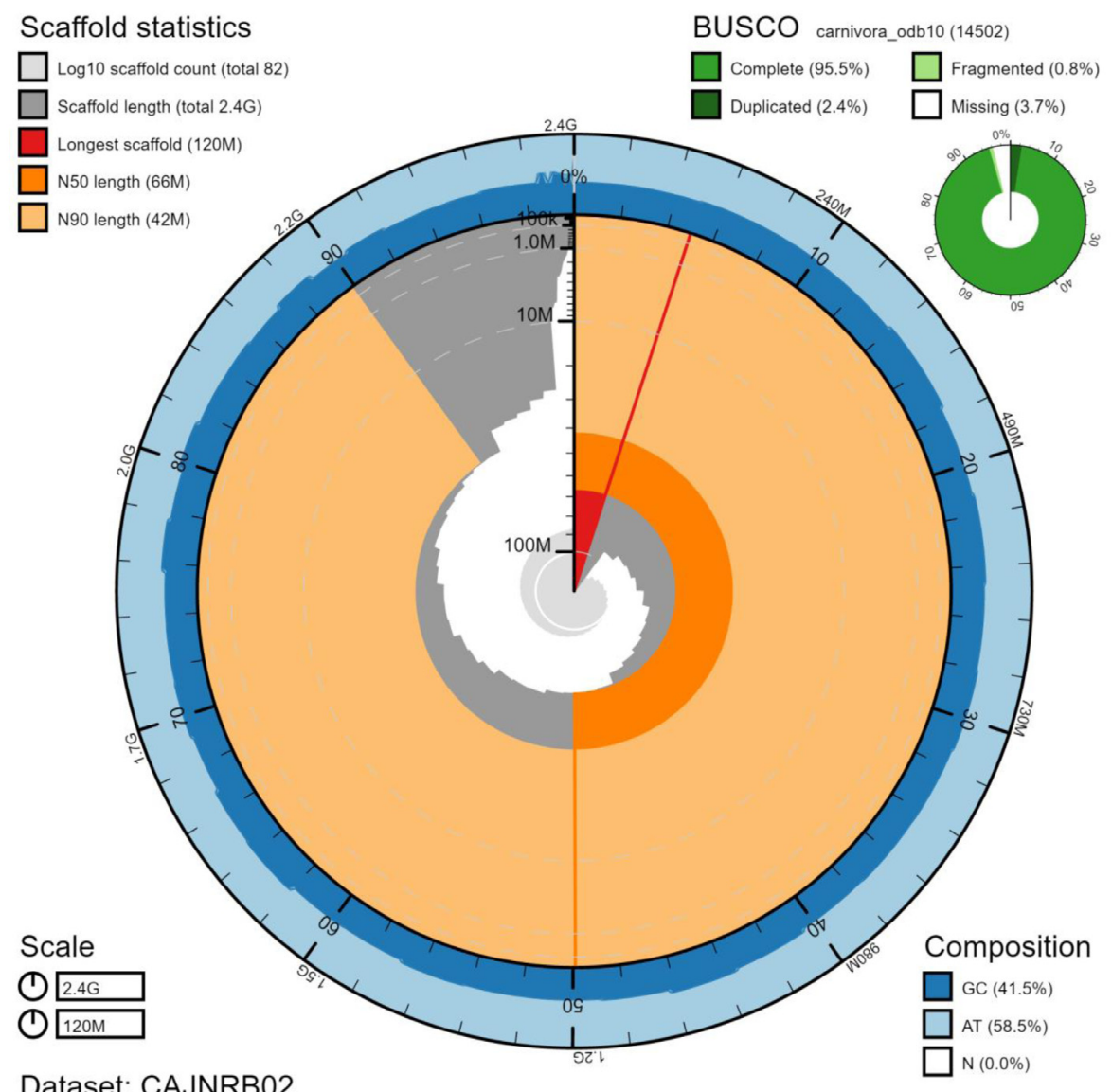

Figure 1. Genome assembly of Canis lupus, mCanLor1.2: metrics. The BlobToolKit Snailplot shows N50 metrics and BUSCO gene completeness. The main plot is divided into 1,000 size-ordered bins around the circumference with each bin representing $0.1 \%$ of the $2,447,463,909 \mathrm{bp}$ assembly. The distribution of scaffold lengths is shown in dark grey with the plot radius scaled to the longest scaffold present in the assembly $(124,665,963$ bp, shown in red). Orange and pale-orange arcs show the N50 and N90 scaffold lengths (65,778,685 and $41,774,919$ bp), respectively. The pale grey spiral shows the cumulative scaffold count on a log scale with white scale lines showing successive orders of magnitude. The blue and pale-blue area around the outside of the plot shows the distribution of GC, AT and N percentages in the same bins as the inner plot. A summary of complete, fragmented, duplicated and missing BUSCO genes in the carnivora_odb10 set is shown in the top right. An interactive version of this figure is available at https://blobtoolkit.genomehubs.org/view/ mCanLor1.2/dataset/CAJNRB02/snail. 


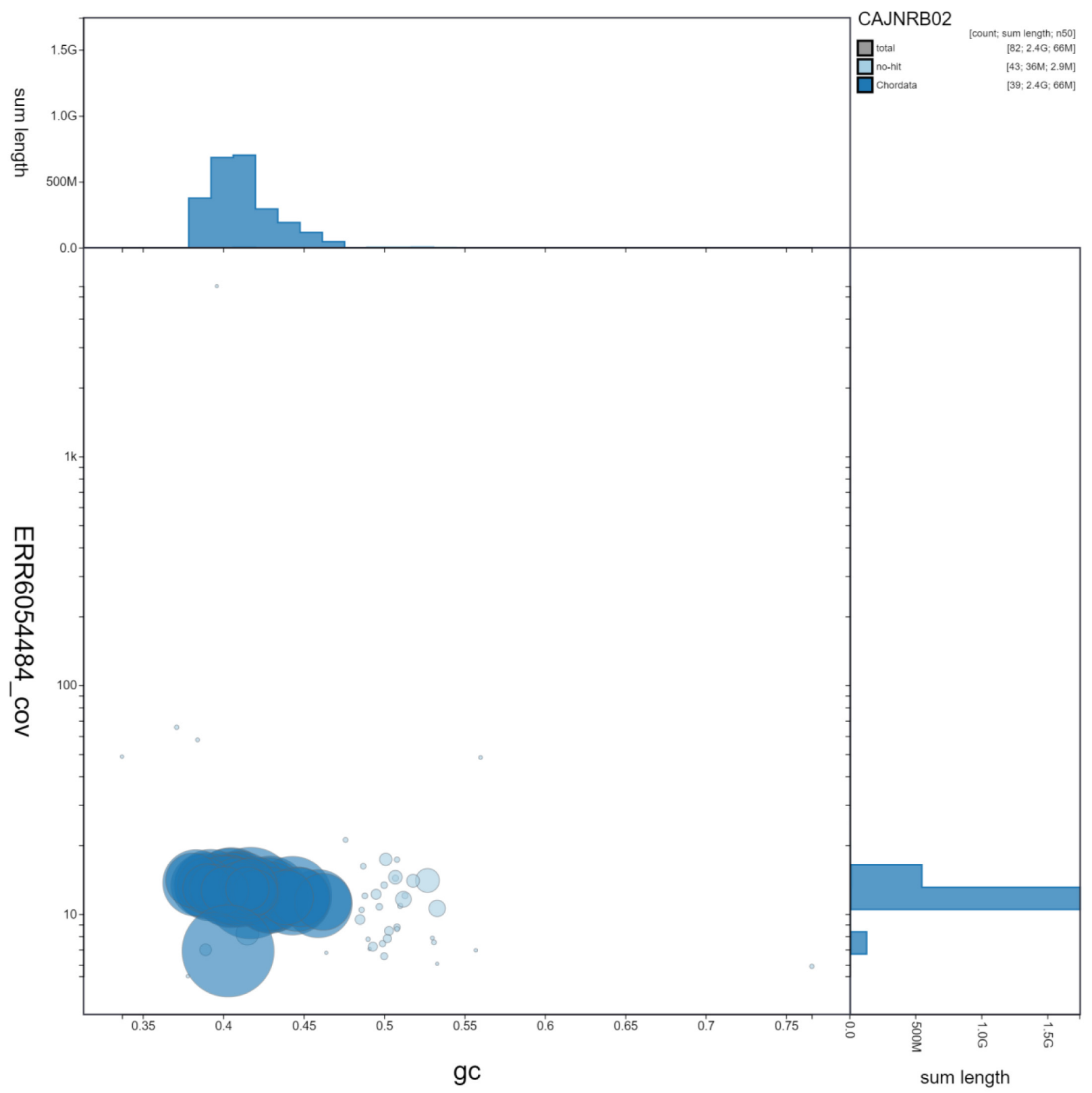

Figure 2. Genome assembly of Canis Iupus, mCanLor1.2: GC coverage. BlobToolKit GC-coverage plot. Scaffolds are coloured by phylum. Circles are sized in proportion to scaffold length. Histograms show the distribution of scaffold length sum along each axis. An interactive version of this figure is available at https://blobtoolkit.genomehubs.org/view/mCanLor1.2/dataset/CAJNRB02/blob.

DNA was extracted from the muscle tissue of mCanLorl at the Wellcome Sanger Institute (WSI) Scientific Operations core from the whole organism using the Qiagen MagAttract HMW DNA kit, according to the manufacturer's instructions. RNA (from the same muscle tissue) was extracted in the Tree of Life Laboratory at the WSI using TRIzol, according to the manufacturer's instructions. RNA was then eluted in $50 \mu \mathrm{l}$ RNAse-free water and its concentration RNA assessed using a Nanodrop spectrophotometer and Qubit Fluorometer using the Qubit RNA Broad-Range (BR) Assay kit. Analysis of the integrity of the RNA was done using Agilent RNA 6000 Pico Kit and Eukaryotic Total RNA assay.
Pacific Biosciences HiFi circular consensus and 10X Genomics read cloud sequencing libraries were constructed according to the manufacturers' instructions. Poly(A) RNA-Seq libraries were constructed using the NEB Ultra II RNA Library Prep kit. DNA sequencing was performed by the Scientific Operations core at the Wellcome Sanger Institute on Pacific Biosciences SEQUEL II and Illumina HiSeq X instruments. RNA sequencing was performed using an Illumina MiSeq instrument. Further 10X sequencing was performed at SciLifeLab, Stockholm, Sweden. DNA was extracted using the automatic KingFisher ${ }^{\mathrm{TM}}$ Duo Prime Purification System (Thermo Fisher Scientific, Bremen, Germany) following the 


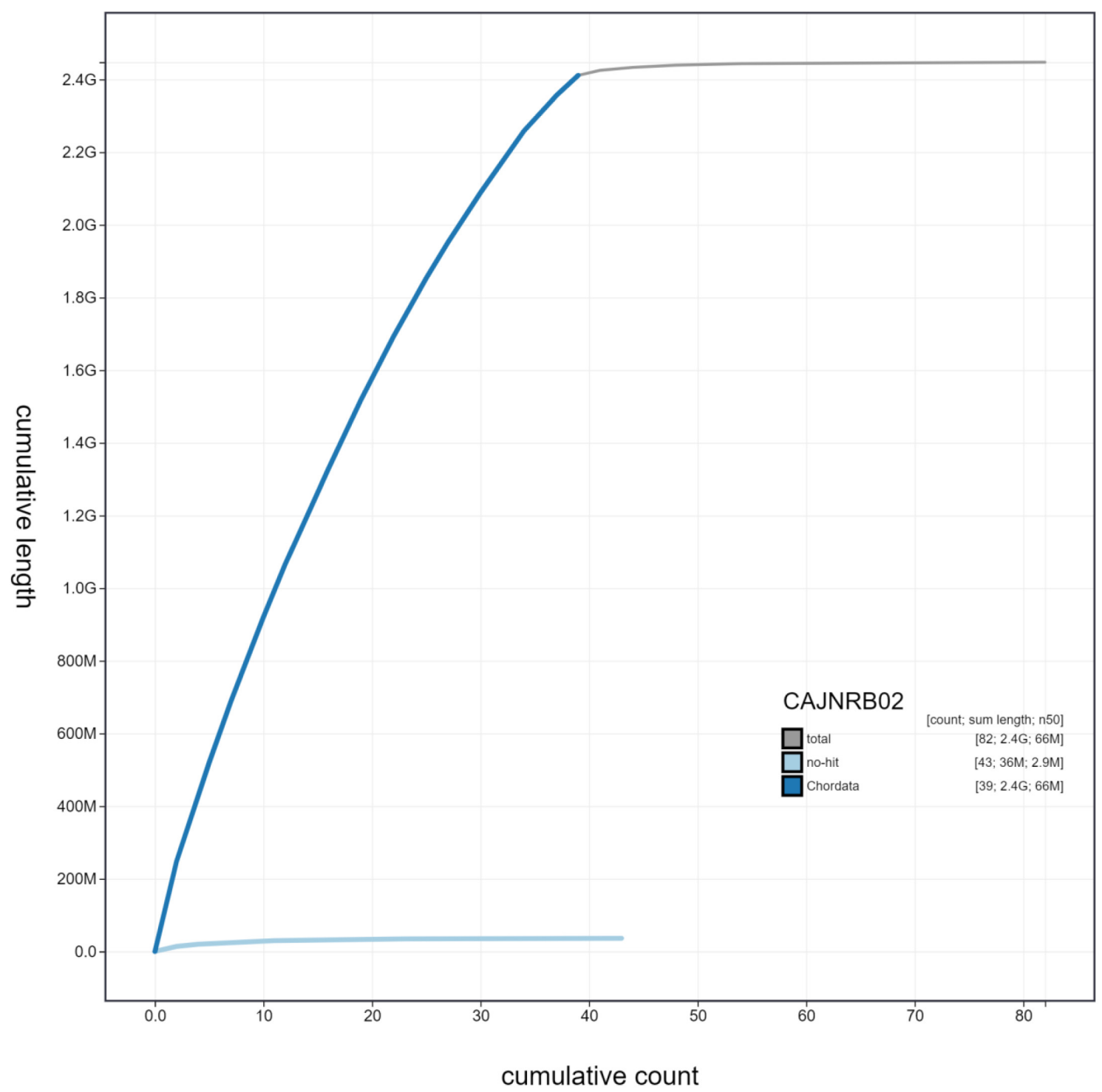

Figure 3. Genome assembly of Canis Iupus, mCanLor1.2: cumulative sequence. BlobToolKit cumulative sequence plot. The grey line shows cumulative length for all scaffolds. Coloured lines show cumulative lengths of scaffolds assigned to each phylum using the buscogenes taxrule. An interactive version of this figure is available at https://blobtoolkit.genomehubs.org/view/mCanLor1.2/dataset/ CAJNRB02/cumulative.

manufacturer's protocol. Following this, Illumina TruSeq PCR-free libraries were constructed and sequencing performed on HiSeq X. Hi-C data were generated at SciLifeLab, Stockholm, Sweden using the Dovetail Hi-C kit and sequenced on HiSeq X.

Assembly was carried out with Hifiasm (Cheng et al., 2021). Haplotypic duplication was identified and removed with purge_dups (Guan et al., 2020). Scaffolding with Hi-C data (Rao et al., 2014) was carried out with SALSA2 (Ghurye et al., 2019). The Hi-C scaffolded assembly was polished with the 10X Genomics Illumina data by aligning to the assembly with longranger align, calling variants with freebayes (Garrison \& Marth, 2012). One round of the Illumina polishing was applied. The mitochondrial genome was assembled with MitoHiFi (Uliano-Silva et al., 2021). The assembly was checked for contamination and corrected using the gEVAL system (Chow et al., 2016) as described previously (Howe et al., 2021). Manual curation (Howe et al., 2021) was performed using gEVAL, HiGlass (Kerpedjiev et al., 2018) and Pretext. Regions of concern were identified and resolved using 10X longranger and genetic mapping data. The genome was analysed within the BlobToolKit environment (Challis et al., 2020). Table 3 contains a list of all software tool versions used, where appropriate. 


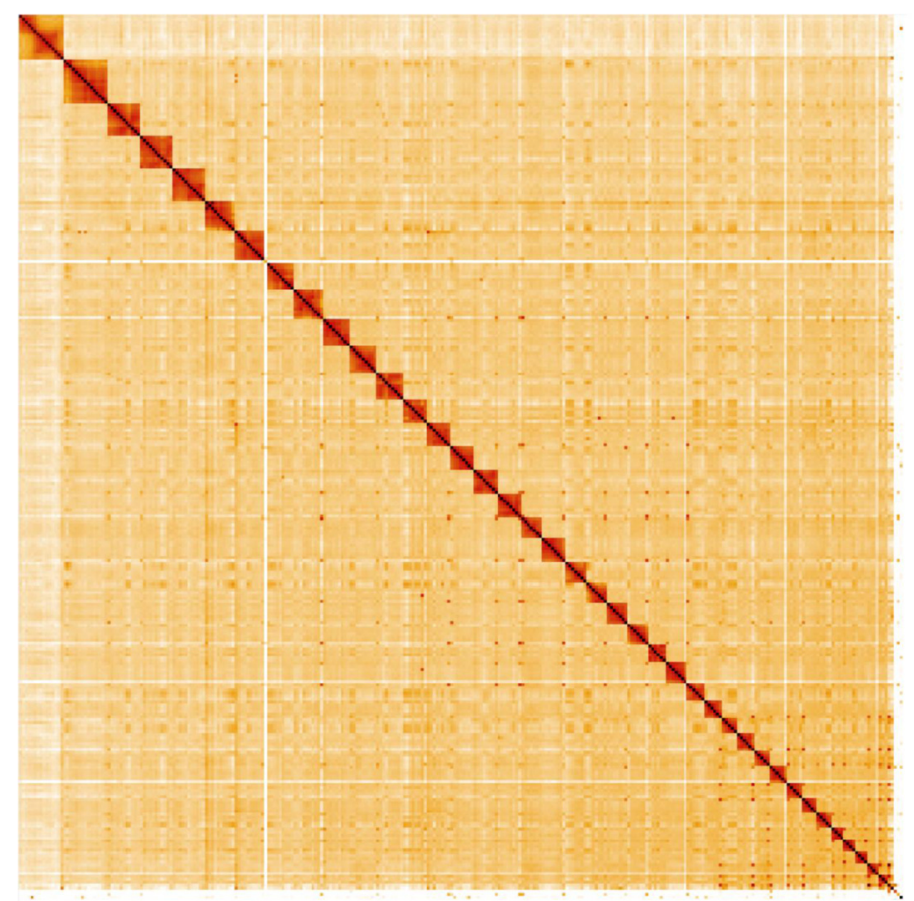

Figure 4. Genome assembly of Canis Iupus, mCanLor1.2: Hi-C contact map. Hi-C contact map of the mCanLor1.2 assembly, visualised in HiGlass. Chromosomes are shown in size order from left to right and top to bottom.

\begin{tabular}{|c|c|c|c|}
\hline INSDC accession & Chromosome & Size (Mb) & GC\% \\
\hline HG994383.1 & 1 & 122.96 & 41.7 \\
\hline HG994387.1 & 2 & 86.40 & 42.9 \\
\hline HG994384.1 & 3 & 93.48 & 40.5 \\
\hline HG994386.1 & 4 & 88.63 & 40.4 \\
\hline HG994385.1 & 5 & 89.78 & 44.3 \\
\hline HG994389.1 & 6 & 78.39 & 42.8 \\
\hline HG994388.1 & 7 & 82.29 & 41.1 \\
\hline HG994390.1 & 8 & 77.59 & 40.8 \\
\hline HG994394.1 & 9 & 66.79 & 45.9 \\
\hline HG994393.1 & 10 & 71.93 & 42.9 \\
\hline HG994391.1 & 11 & 75.75 & 40.4 \\
\hline HG994392.1 & 12 & 73.73 & 39.2 \\
\hline HG994397.1 & 13 & 65.44 & 40.3 \\
\hline HG994400.1 & 14 & 62.79 & 39 \\
\hline HG994395.1 & 15 & 65.78 & 40.5 \\
\hline HG994398.1 & 16 & 63.67 & 41.5 \\
\hline HG994396.1 & 17 & 65.96 & 41.9 \\
\hline HG994402.1 & 18 & 57.59 & 43 \\
\hline HG994403.1 & 19 & 56.75 & 38.7 \\
\hline HG994401.1 & 20 & 59.77 & 44.6 \\
\hline
\end{tabular}

\begin{tabular}{|c|c|c|c|}
\hline INSDC accession & Chromosome & Size (Mb) & GC\% \\
\hline HG994405.1 & 21 & 53.11 & 40.4 \\
\hline HG994399.1 & 22 & 63.45 & 38.3 \\
\hline HG994406.1 & 23 & 52.96 & 40 \\
\hline HG994407.1 & 24 & 49.88 & 44.7 \\
\hline HG994404.1 & 25 & 53.62 & 41.6 \\
\hline HG994409.1 & 26 & 46.11 & 46.2 \\
\hline HG994408.1 & 27 & 48.75 & 40.8 \\
\hline HG994413.1 & 28 & 42.48 & 43.9 \\
\hline HG994412.1 & 29 & 44.09 & 38.9 \\
\hline HG994415.1 & 30 & 41.62 & 41.6 \\
\hline HG994411.1 & 31 & 44.76 & 41 \\
\hline HG994414.1 & 32 & 41.77 & 38.1 \\
\hline HG994417.1 & 33 & 32.66 & 39.4 \\
\hline HG994410.1 & 34 & 45.90 & 41.6 \\
\hline HG994419.1 & 35 & 28.53 & 42 \\
\hline HG994416.1 & 36 & 33.43 & 39 \\
\hline HG994418.1 & 37 & 31.50 & 40.1 \\
\hline HG994420.1 & 38 & 26.44 & 41.5 \\
\hline HG994381.1 & $X$ & 124.67 & 40.3 \\
\hline HG994382.1 & $Y$ & 6.54 & 41.5 \\
\hline HG998573.1 & MT & 0.02 & 39.6 \\
\hline- & Unplaced & 29.74 & 50.3 \\
\hline
\end{tabular}


Table 3. Software tools used.

\begin{tabular}{|l|l|l|}
\hline Software tool & Version & Source \\
\hline Hifiasm & 0.12 & Cheng et al., 2021 \\
\hline purge_dups & 1.2 .3 & Guan et al., 2020 \\
\hline SALSA2 & 2.2 & Ghurye et al., 2019 \\
\hline longranger align & 2.2 .2 & $\begin{array}{l}\text { https://support.10xgenomics.com/ } \\
\text { genome-exome/software/pipelines/latest/ } \\
\text { advanced/other-pipelines }\end{array}$ \\
\hline freebayes & $1.3 .1-17-g a a 2 a c e 8$ & Garrison \& Marth, 2012 \\
\hline MitoHiFi & 1 & Uliano-Silva et al., 2021 \\
\hline gEVAL & N/A & Chow et al., 2016 \\
\hline PretextView & $0.1 . x$ & https://github.com/wtsi-hpag/PretextView \\
\hline HiGlass & 1.11 .6 & Kerpedjiev et al., 2018 \\
\hline BlobToolKit & 2.6 .2 & Challis et al., 2020 \\
\hline
\end{tabular}

\section{Data availability}

European Nucleotide Archive: Canis lupus (Greenland wolf). Accession number PRJEB43200; https://identifiers.org/ena.embl/ PRJEB43200.

The genome sequence is released openly for reuse. The C. lupus genome sequencing initiative is part of the Darwin Tree of Life (DToL) project and the Vertebrate Genomes Project. All raw sequence data and the assembly have been deposited in INSDC databases. The genome will be annotated using the RNA-Seq data and presented through the Ensembl pipelineat the European Bioinformatics Institute. Raw data and assembly accession identifiers are reported in Table 1.
Author information

Members of the Darwin Tree of Life Barcoding collective are listed here: https://doi.org/10.5281/zenodo.4893704.

Members of the Wellcome Sanger Institute Tree of Life programme are listed here: https://doi.org/10.5281/zenodo. 5377053.

Members of Wellcome Sanger Institute Scientific Operations: DNA Pipelines collective are listed here: https://doi.org/10.5281/ zenodo.4790456.

Members of the Tree of Life Core Informatics collective are listed here: https://doi.org/10.5281/zenodo.5013542.

Members of the Darwin Tree of Life Consortium are listed here: https://doi.org/10.5281/zenodo.4783559.
Challis R, Richards E, Rajan J, et al.: BlobToolKit - Interactive Quality Assessment of Genome Assemblies. G3 (Bethesda). 2020; 10(4): 1361-74. PubMed Abstract | Publisher Full Text | Free Full Text

Cheng $\mathrm{H}$, Concepcion GT, Feng X, et al:: Haplotype-Resolved de Novo Assembly Using Phased Assembly Graphs with Hifiasm. Nat Methods. 2021 18(2): 170-75.

PubMed Abstract | Publisher Full Text | Free Full Text

Chow W, Brugger K, Caccamo M, et al.: gEVAL - a web-based browser for evaluating genome assemblies. Bioinformatics. 2016; 32(16): 2508-10. PubMed Abstract | Publisher Full Text | Free Full Text

Dawes PR, Elander M, Ericson M: The Wolf (Canis Lupus) in Greenland: A Historical Review and Present Status. Arctic. 1986; 39(2): 119-32. Publisher Full Text

Garrison E, Marth G: Haplotype-Based Variant Detection from Short-Read Sequencing. arXiv: 1207.3907. 2012.

Reference Source

Ghurye J, Rhie A, Walenz BP, et al.: Integrating Hi-C Links with Assembly
Graphs for Chromosome-Scale Assembly. PLoS Comput Biol. 2019; 15(8): e1007273.

PubMed Abstract | Publisher Full Text | Free Full Text

Gopalakrishnan S, Castruita JAS, Sinding MS, et al:: The Wolf Reference Genome Sequence (Canis Lupus Lupus) and Its Implications for Canis Spp. Population Genomics. BMC Genomics. 2017; 18(1): 495.

PubMed Abstract | Publisher Full Text | Free Full Text

Gopalakrishnan S, Sinding MS, Ramos-Madrigal J, et al.: Interspecific Gene Flow Shaped the Evolution of the Genus Canis. Curr Biol. 2019; 29(23): 4152 PubMed Abstract | Publisher Full Text | Free Full Text

Guan D, McCarthy SA, Wood J, et al.: Identifying and Removing Haplotypic Duplication in Primary Genome Assemblies. Bioinformatics. 2020; 36(9): 2896-98.

PubMed Abstract | Publisher Full Text | Free Full Text

Howe K, Chow W, Collins J, et al:: Significantly Improving the Quality of Genome Assemblies through Curation. Gigascience. 2021; 10(1): giaa153. PubMed Abstract | Publisher Full Text | Free Full Text 
Jhala Y, Boitani L, Phillips M: IUCN Red List of Threatened Species: Canis Lupus. IUCN Red List of Threatened Species. 2018.

Publisher Full Text

Kerpedjiev P, Abdennur N, Lekschas F, et al:: HiGlass: Web-Based Visual Exploration and Analysis of Genome Interaction Maps. Genome Biol. 2018; 19(1): 125.

PubMed Abstract | Publisher Full Text | Free Full Text

Marquard-Petersen U: Invasion of Eastern Greenland by the High Arctic Wolf Canis Lupus Arctos. Wildl Biol. 2011; 17(4): 383-88.

Publisher Full Text

Marquard-Petersen U: Decline and Extermination of an Arctic Wolf Population in East Greenland, 1899-1939. Arctic. 2012; 65(2): 121-243. Publisher Full Text

Marquard-Petersen U: Sudden Death of an Arctic Wolf Population in

Greenland. Polar Res. 2021; 40

Publisher Full Text

Nilsen EB, Milner-Gulland EJ, Schofield L, et al.: Wolf Reintroduction to Scotland: Public Attitudes and Consequences for Red Deer Management. Proc Biol Sci. 2007; 274(1612): 995-1002.

PubMed Abstract | Publisher Full Text | Free Full Text

Rao SSP, Huntley MH, Durand NC, et al.: A 3D Map of the Human Genome at Kilobase Resolution Reveals Principles of Chromatin Looping. Cell. 2014; 159(7): 1665-80.

PubMed Abstract | Publisher Full Text | Free Full Text

Ripple WJ, Beschta RL: Trophic cascades in Yellowstone: The first 15 years after wolf reintroduction. Biol Conserv. 2012; 145(1): 205-13.

Publisher Full Text

Ripple WJ, Estes JA, Beschta RL, et al.: Status and ecological effects of the world's largest carnivores. Science. 2014; 343(6167): 1241484

PubMed Abstract | Publisher Full Text

Simão FA, Waterhouse RM, Ioannidis P, et al: BUSCO: Assessing Genome Assembly and Annotation Completeness with Single-Copy Orthologs. Bioinformatics. 2015. 31(19): 3210-12.

PubMed Abstract | Publisher Full Text

Sinding MS, Gopalakrishan S, Vieira FG, et al.: Population Genomics of Grey Wolves and Wolf-like Canids in North America. PLOS Genet. 2018; 14(11): e1007745.

PubMed Abstract | Publisher Full Text | Free Full Text

Skoglund P, Ersmark E, Palkopoulou E, et al:: Ancient Wolf Genome Reveals an Early Divergence of Domestic Dog Ancestors and Admixture into HighLatitude Breeds. Curr Biol. 2015; 25(11): 1515-19.

PubMed Abstract | Publisher Full Text

Uliano-Silva M, Nunes JGF, Krasheninnikova K, et al.: marcelauliano/MitoHiFi: mitohifi_v2.0. 2021.

Publisher Full Text

Wang GD, Zhai W, Yang HC, et al: O Out of Southern East Asia: The Natural History of Domestic Dogs across the World. Cell Res. 2016; 26(1): 21-33. PubMed Abstract | Publisher Full Text | Free Full Text

Wilson Cl: Could We Live with Reintroduced Large Carnivores in the UK? Mamm Rev. 2004; 34(3): 211-32.

Publisher Full Text 


\title{
Open Peer Review
}

\section{Current Peer Review Status:}

\section{Version 1}

Reviewer Report 06 December 2021

https://doi.org/10.21956/wellcomeopenres.19162.r47083

(C) 2021 Allio R. This is an open access peer review report distributed under the terms of the Creative Commons Attribution License, which permits unrestricted use, distribution, and reproduction in any medium, provided the original work is properly cited.

\section{Rémi Allio}

Montpellier Institute of Evolutionary Sciences (ISEM), CNRS, IRD, EPHE, University of Montpellier, Montpellier, France

The Data Note "The genome sequence of the grey wolf, Canis lupus Linnaeus 1758" presented by Sinding et al. is the description of the genome of the grey wolf obtained following the Darwin Tree of Life protocols. PacBio single-molecule long reads, 10X Genomics read clouds, Illumina reads and $\mathrm{Hi}-\mathrm{C}$ data were generated and used to assemble this genome. RNA-seq libraries were also constructed and sequenced. However, the authors do not elaborate on why and where these are used (for Ensembl pipeline?).

The resulting assembly is of high quality with the majority of the assembly ( $99 \%)$ scaffolded into 40 chromosomal pseudomolecules.

However, given the genome provided here, I think a comparison (based on BUCSO scores or traditional measures such as number of scaffolds or N50) with other genomes available for the species Canis lupus would have been interesting. Indeed, even if this genome is the first available assembly for the subspecies Canis lupus orion, there are 25 other assemblies for this species available on NCBI, with among them, other chromosome length genome assemblies.

Minor but mandatory comments:

Explain the use of RNA-seq data. Is it only for use in the Ensembl pipeline?

Not all softwares are presented in Table 3. At least BUSCO and its version are missing.

\author{
Is the rationale for creating the dataset(s) clearly described? \\ Yes
}

Are the protocols appropriate and is the work technically sound?

Yes 
Are sufficient details of methods and materials provided to allow replication by others? Yes

Are the datasets clearly presented in a useable and accessible format?

Yes

Competing Interests: No competing interests were disclosed.

Reviewer Expertise: Phylogenomics and Genomics

I confirm that I have read this submission and believe that I have an appropriate level of expertise to confirm that it is of an acceptable scientific standard.

Reviewer Report 25 November 2021

\section{https://doi.org/10.21956/wellcomeopenres.19162.r47087}

(C) 2021 Hiller $\mathbf{M}$. This is an open access peer review report distributed under the terms of the Creative Commons Attribution License, which permits unrestricted use, distribution, and reproduction in any medium, provided the original work is properly cited.

\section{Michael Hiller}

1 LOEWE Centre for Translational Biodiversity Genomics, Frankfurt am Main, Germany

2 Senckenberg Society for Nature Research, Frankfurt am Main, Germany

3 Goethe University, Frankfurt am Main, Germany

The publication describes a high-quality genome of the Polar wolf, which will be an asset for population genomics, pangenomics of Canids and likely research aiming at understanding dog domestication. The study is well motivated, the methods are clearly described and the assembly is done by an expert team of genome scientists.

I have only two suggestions:

1. The method section mentions that HiFi circular consensus reads were produced. Maybe this can be mentioned above in the genome report:

"A total of 28-fold coverage in Pacific Biosciences single-molecule, circular consensus (HiFi) long reads ..."

2. Since $10 X$ data was also produced and used for polishing, it makes sense to use Merqury to compute a base QV value. Maybe this can be added.

Is the rationale for creating the dataset(s) clearly described?

Yes

Are the protocols appropriate and is the work technically sound?

Yes 
Are sufficient details of methods and materials provided to allow replication by others? Yes

Are the datasets clearly presented in a useable and accessible format?

Yes

Competing Interests: No competing interests were disclosed.

Reviewer Expertise: genomics

I confirm that I have read this submission and believe that I have an appropriate level of expertise to confirm that it is of an acceptable scientific standard. 